\title{
Erroneously High Lactate: A Guide to Diagnosing Ethylene Glycol Poisoning
}

\section{Mayanka Kamboj Harini Bejjanki Saraswathi Gopal Rupam Ruchi}

Division of Nephrology, Hypertension, and Renal Transplantation, University of Florida, Gainesville, FL, USA

\section{What Is It About?}

Ethylene glycol (EG) intoxication is a medical emergency. In the absence of specific signs and symptoms, the diagnosis can be challenging as the methods for directly measuring EG metabolites are available at limited laboratories. Spuriously high lactic acid levels can be seen due to analytical interference in lactic acid measurement in the presence of EG metabolites. Discovery of a lactate gap using different laboratory techniques can serve as an important clue for early diagnoses of EG intoxication, especially if clinical history is not available.

\section{Keywords}

Ethylene glycol poisoning · Lactic acidosis · Analytical interference · Toxicology

\section{Abstract}

A high lactic acid level in critically ill patients is a marker of poor prognosis. However, lactic acidosis in ethylene glycol (EG) poisoning should be interpreted cautiously as analytical interference is observed with EG metabolites. 


\section{Established Facts}

- Small lactic acidosis is frequently seen in ethylene glycol poisoning.

- Ethylene glycol metabolites can cause analytical interference with lactic acid measurement.

\section{Novel Insights}

- Understanding specific laboratory methods where the analytical interference is observed can assist clinicians in discovering the underlying etiology of unexplained lactic acidosis.

- The identification of a lactate gap using different laboratory methods can assist in making the diagnoses of ethylene glycol poisoning.

\section{Introduction}

Lactic acidosis, a common cause of metabolic acidosis in critically ill patients, often occurs secondary to overt lactate production or decreased clearance. However, factitious elevation of lactate levels can be seen in ethylene glycol (EG) poisoning.

\section{Case Report}

A 47-year-old man was brought to the emergency room with altered mental status. He was afebrile, somnolent, and normotensive. Lab work showed sodium $146 \mathrm{mmol} / \mathrm{L} \mathrm{HCO}_{3}{ }^{-}$ $11 \mathrm{mmol} / \mathrm{L}$, anion gap (AG) 24, creatinine $1.4 \mathrm{mg} / \mathrm{dL}$, serum osmolality $310 \mathrm{mosm} / \mathrm{kg}$, osmolal gap $6 \mathrm{mosm} / \mathrm{Kg}$, whole-blood lactic acid (LA) $11 \mathrm{mmol} / \mathrm{L}$, venous blood gas $\mathrm{pH} 7.2$, and $\mathrm{pCO}_{2}$ $33 \mathrm{~mm} \mathrm{Hg}$. Toxicology screen was negative for alcohol, acetaminophen, and salicylates. He reported consuming anti-freeze approximately $18 \mathrm{~h}$ prior to presentation. Lab error in LA measurement was suspected. Repeat whole-blood LA was $10.3 \mathrm{mmol} / \mathrm{L}$, and simultaneously drawn plasma LA was $3 \mathrm{mmol} / \mathrm{L}$. The patient received IV fomepizole, and hemodialysis was initiated for management of EG toxicity. Over the next $24 \mathrm{~h}$, AG metabolic acidosis resolved. His serum creatinine peaked to $1.8 \mathrm{mg} / \mathrm{dL}$ over the next 2 days and improved to $1.4 \mathrm{mg} / \mathrm{dL}$ on hospital discharge.

\section{Results}

Whole-blood LA was measured using the cartridge-based blood gas analyzer ABL800 flex. It utilizes the amperometric method to measure the concentration of lactate oxidized at the electrode which is proportional to the electrical current flowing through an electrode chain. Due to a lack of specificity of lactate oxidase, analytical interference in the presence of glycolic acid has been reported [1]. Plasma LA was measured with the Abbott Architect ci8200 intergrated analyzer system. It utilizes the quantitative enzymatic method. Lactate is converted to pyruvate and hydrogen peroxide $\left(\mathrm{H}_{2} \mathrm{O}_{2}\right)$. The $\mathrm{H}_{2} \mathrm{O}_{2}$ oxidizes a chromogen precursor to produce a colored dye. The increase in absorbance at $548 \mathrm{~nm}$ is proportional to the LA concentration in the sample. No analytic interference in LA measurement is seen in the presence of EG metabolites with this method. 


\section{Discussion/Conclusion}

EG is rapidly absorbed in the gastrointestinal tract after ingestion. It is metabolized utilizing alcohol dehydrogenase and aldehyde dehydrogenase to glycolaldehyde, glycolic acid/glycolate, and eventually oxalic acid. Oxalic acid binds to calcium to form calcium oxalate crystals which can cause acute kidney injury. A high osmolal gap with normal AG is seen in the early phase of ingestion; however, after conversion of EG to its toxic metabolites in the late phase of ingestion, a normal osmolal gap with high AG and calcium oxalate crystals are seen. The diagnosis of EG poisoning can be difficult to establish in the absence of clinical history as these biochemical abnormalities are nonspecific and methods which directly measure EG levels are only available at limited reference laboratories. A small elevation in LA can be seen with EG poisoning. However, erroneously high LA has been reported with EG poisoning due to the use of lab instruments which cannot differentiate between lactate and glycolate because of their structural similarity. A true elevation of the LA level can be seen with lactate overproduction or decreased clearance in the setting of tissue hypoperfusion, liver disease, inborn errors of metabolism, and drug or toxin exposure. High LA seen in critically ill patients has been associated with increased mortality and morbidity [2]. In our patient, increased AG was explained with increased LA; however, no apparent cause was found for lactic acidosis. Unexplained high LA should be confirmed with a second reference test which uses different methodology. Discovery of a lactate gap using 2 different methodologies of LA measurement can help differentiate EG poisoning from other causes of lactic acidosis. Furthermore, a lactate gap should raise suspicion for EG ingestion, especially if the clinical history is not available.

\section{Statement of Ethics}

The authors have no ethical conflicts to disclose.

\section{Disclosure Statement}

The authors have no potential conflict of interest to declare with respect to this paper.

\section{Funding Sources}

No specific financial support was obtained for the preparation of this article.

\section{Author Contributions}

Mayanka Kamboj: Drafting manuscript and data collection. Harini Bejjanki: Drafting manuscript and data collection. Saraswathi Gopal: Revising the article critically for important intellectual content. Rupam Ruchi: Revising the article critically for important intellectual content.

\section{References}

1 Radiometer Medical ApS. ABL800 FLEX Reference manual [Internet]. Denmark [cited 2004 Dec 2004]. Available from: http://www3.hscni.net/stlabs/webhb/poct/documents/poct\%20abl800\%20man.pdf.

2 Seheult J, Fitzpatrick G, Boran G. Lactic acidosis: an update. Clin Chem Lab Med. 2017 Mar;55(3):322-33. 